\title{
Dynamic response of a strongly perturbed electron gas
}

\author{
R. Díez Muiño, ${ }^{1}$ A. Arnau, ${ }^{2}$ A. Salin, ${ }^{1,3}$ and P. M. Echenique ${ }^{1,2}$ \\ ${ }^{1}$ Donostia International Physics Center (DIPC), P. Manuel de Lardizabal 4, 20018 San Sebastián, Spain \\ ${ }^{2}$ Departamento de Física de Materiales, Facultad de Químicas UPV/EHU, and Unidad de Física de Materiales, \\ Centro Mixto CSIC-UPV/EHU, Apartado 1072, 20080 San Sebastián, Spain \\ ${ }^{3}$ Laboratoire de Physico-Chimie Moléculaire, UMR 5803 CNRS-Université de Bordeaux I, 351 Cours de la Libération, \\ 33405 Talence Cedex, France
}

(Received 16 May 2003; published 30 July 2003)

\begin{abstract}
The dynamic response of an electron gas strongly perturbed by an impurity is calculated using density functional theory and many-body linear response theory. We find that the piling up of charge induced by the impurity significantly modifies electron dynamics in the vicinity of the impurity and the response of the system to localized external perturbations. Our results show the important local effects that the nonlinear screening of impurities introduce in the dynamic response of metallic systems.
\end{abstract}

DOI: 10.1103/PhysRevB.68.041102 PACS number(s): 71.15.Mb, 71.10.Li, 78.70.-g, 79.20.Fv

The theoretical description of many-body systems in their ground state has nowadays become an affordable task because of recent advances in the numerical implementation of $a b$ initio methods, as well as huge improvements in computing capabilities. However, an accurate evaluation of excitedstates properties in such systems is still a major theoretical challenge. The problem is of obvious importance as most experimental techniques are based on testing of excited state. Apart from its intrinsic fundamental interest, the knowledge of the system excitation spectrum is useful to understand a large variety of physical phenomena, such as (i) photoabsorption of atoms ${ }^{1,2}$ and plasmas, ${ }^{3,4}$ (ii) optical response of clusters, ${ }^{5-8}$ (iii) core polarization and dielectric response in solids, ${ }^{9,10}$ (iv) resonant photoemission, ${ }^{11}$ or (v) neutralization of ions at surfaces. ${ }^{12,13}$

Common solid-state approaches to study excited states are many-body perturbation and time-dependent densityfunctional theories. The application of these methods to real systems often relies on system symmetries, such as twodimensional or three-dimensional periodicity. The theoretical description of electronic excitations turns out to be more intricate when a localized impurity breaks the spatial symmetry. Numerical methods based on system periodicity are not efficient in this case. Furthermore, localized perturbations in solids, such as impurities in bulk, inner-shell holes, or adsorbates at surfaces strongly modify their environment. The appearance of new states bound to the impurity ${ }^{14}$ and/or resonances in the continuum ${ }^{15}$ can change dramatically the local properties of the system. The influence of impurity atoms in the excitation spectrum of a $2 \mathrm{D}$ electron gas (where any attractive potential has a bound state) has been considered recently. ${ }^{16}$ Kondo-type effects arise for single magnetic impurities as well. ${ }^{17,18}$

In metallic systems, valence-band electrons are highly mobile and the presence of an atomic impurity introduces a strong distortion in the electronic density. Our purpose in this work is to show that this rearrangement of electronic charge determines the local dynamic response of the valence electrons to external perturbations. We study the case of an atomic impurity embedded in a free electron gas (FEG), which represents the valence band of a simple metal (such as
$\mathrm{Na}$ or $\mathrm{Al}$ ). Therefore, band-structure effects are not considered as we are interested in determining the local dynamic response of the system. We use density-functional theory to calculate the ground state of the system and obtain the valence-electron response function in linear theory afterwards. We show that the dynamic screening of the Coulomb interaction is highly modified in the vicinity of the impurity and has a complex behavior determined by two competing effects. As an example, we calculate transition probabilities for hole-filling processes in the embedded impurity. We compare some of our results with those obtained in calculations which do not include the presence of the impurity to remark the specific effects introduced by the latter.

For small external dynamic perturbations of the system, the many-body response is well described by the linear density response function $\chi\left(\mathbf{r}, \mathbf{r}^{\prime}, \omega\right)$. The self-consistent response function $\chi\left(\mathbf{r}, \mathbf{r}^{\prime}, \omega\right)$ can be obtained at the randomphase approximation (RPA) level in terms of the response function of a system of independent particles $\chi^{0}\left(\mathbf{r}, \mathbf{r}^{\prime}, \omega\right)$ (atomic units are used throughout unless otherwise stated):

$$
\begin{aligned}
\chi\left(\mathbf{r}, \mathbf{r}^{\prime}, \omega\right)= & \chi^{0}\left(\mathbf{r}, \mathbf{r}^{\prime}, \omega\right) \\
& +\int d \mathbf{r}_{1} d \mathbf{r}_{2} \chi^{0}\left(\mathbf{r}, \mathbf{r}_{1}, \omega\right) V_{\mathrm{C}}\left(\mathbf{r}_{1}, \mathbf{r}_{2}\right) \chi\left(\mathbf{r}_{2}, \mathbf{r}^{\prime}, \omega\right),
\end{aligned}
$$

where $V_{\mathrm{C}}\left(\mathbf{r}_{1}, \mathbf{r}_{2}\right)$ is the Coulomb interaction, and $\chi^{0}\left(\mathbf{r}, \mathbf{r}^{\prime}, \omega\right)$ is given by ${ }^{1,2}$

$$
\begin{aligned}
\chi^{0}\left(\mathbf{r}, \mathbf{r}^{\prime}, \omega\right)= & \sum_{i \in o c c}\left[\psi_{i}^{*}(\mathbf{r}) \psi_{i}\left(\mathbf{r}^{\prime}\right) G\left(\mathbf{r}, \mathbf{r}^{\prime}, \epsilon_{i}+\omega\right)\right. \\
& \left.+\psi_{i}(\mathbf{r}) \psi_{i}^{*}\left(\mathbf{r}^{\prime}\right) G^{*}\left(\mathbf{r}, \mathbf{r}^{\prime}, \boldsymbol{\epsilon}_{i}-\omega\right)\right]
\end{aligned}
$$

We study the valence-electrons response. For this reason, the sum over $i$ in Eq. (2) runs over occupied states in the valence band, and we use a modified one-electron Green's function $G\left(\mathbf{r}, \mathbf{r}^{\prime}, \omega\right)$ in which the core contributions are explicitly subtracted. ${ }^{9} \psi_{i}(\mathbf{r})$ and $\epsilon_{i}$ are respectively the oneelectron wave functions and energy levels that are eigenstates of the Kohn-Sham (KS) Hamiltonian $H=-\nabla^{2} / 2$ 
$+V_{\text {eff }} .{ }^{19}$ The effective potential in the latter equation includes several terms: $V_{\text {eff }}=V_{\text {Hartree }}+V_{\text {xc }}+V_{\text {imp }} \cdot V_{\text {Hartree }}$ is the electrostatic potential and $V_{\mathrm{xc}}$ is the exchange correlation potential, which is calculated in the local density approximation. ${ }^{20}$ The essential point of our work is that we use the full Hamiltonian that includes $V_{\text {imp }}$ in $V_{\text {eff }}$ to explicitly account for the presence of the impurity in the calculation of the system response.

Our numerical procedure is the following: we solve the KS one-electron equations after expansion of the wave functions in a spherical harmonic basis set $Y_{l, m}(\Omega)$. The oneelectron Green's functions $G\left(\mathbf{r}, \mathbf{r}^{\prime}, \omega\right)$ are also built from the solutions of the KS equation for every $\omega .^{1,2}$ With these ingredients we calculate the multipole components of $\chi^{0}\left(\mathbf{r}, \mathbf{r}^{\prime}, \omega\right)$. Integral equation (1) is solved in real space for every polar component of $\chi\left(\mathbf{r}, \mathbf{r}^{\prime}, \omega\right)$.

Electron dynamics is characterized by the screened Coulomb interaction $W\left(\mathbf{r}, \mathbf{r}^{\prime}, \omega\right) .{ }^{21}$ In RPA, $W\left(\mathbf{r}, \mathbf{r}^{\prime}, \omega\right)$ is defined in terms of $V_{\mathrm{C}}$ as

$$
\begin{aligned}
W\left(\mathbf{r}, \mathbf{r}^{\prime}, \omega\right)= & V_{\mathrm{C}}\left(\mathbf{r}, \mathbf{r}^{\prime}\right) \\
& +\int d \mathbf{r}_{1} d \mathbf{r}_{2} V_{\mathrm{C}}\left(\mathbf{r}, \mathbf{r}_{1}\right) \chi\left(\mathbf{r}_{1}, \mathbf{r}_{2}, \omega\right) V_{\mathrm{C}}\left(\mathbf{r}_{2}, \mathbf{r}^{\prime}\right) .
\end{aligned}
$$

The probability of inelastic processes, determined by the imaginary part of the screened interaction Im $\left[-W\left(\mathbf{r}, \mathbf{r}^{\prime}, \omega\right)\right]$, is shown in the lower panels of Fig. 1 for the case of an Ar atom embedded in a FEG of $r_{s}=2\left(r_{s}\right.$ is defined from $n_{0}=3 / 4 \pi r_{s}^{3}$, where $n_{0}$ is the electronic density of the gas). The OX axis is defined as the line that joins the position of the Ar (the origin of coordinates) and the position of the Coulomb source $x$. We show a case in the low-energy range $\omega \approx 0.17 \omega_{p}$ (with $\omega_{p}^{2}=4 \pi n_{0}$ ). Two results for Im $\left[-W\left(x, x^{\prime}, \omega\right)\right]$ are shown in the plot: one using the Lindhard response function (plane wave states), and a second one using the self-consistent response that includes the $\mathrm{Ar}$ atom. In both cases the absolute value of Im $\left[-W\left(x, x^{\prime}, \omega\right)\right]$ reaches its maximum at approximately $x$ $=x^{\prime}$. However, there are substantial differences between both calculations in the vicinity of the $\mathrm{Ar}$ atom. Im $\left[-W\left(x, x^{\prime}, \omega\right)\right]$ is affected by the rearrangement of electronic charge induced by the Ar atom in the valence band $\Delta n$. The latter is localized in a region of few atomic units, as shown in the upper panel of Fig. 1. The screening provided by $\Delta n$ reduces the interaction strength significantly and introduces some structure in the shape of $\operatorname{Im}\left[-W\left(x, x^{\prime}, \omega\right)\right]$. Therefore, electron-excitation processes taking place in the neighborhood of the Ar impurity are influenced by this effect. For larger values of $x$, corresponding to a Coulomb source farther from the Ar atom $(x \approx 5$, where total screening of the impurity is already achieved), the unperturbed FEG result is practically recovered.

It is worth to mention that the breakdown of symmetry introduced by the Ar atom is essentially different from that introduced by a surface: in the latter, the electronic excita-

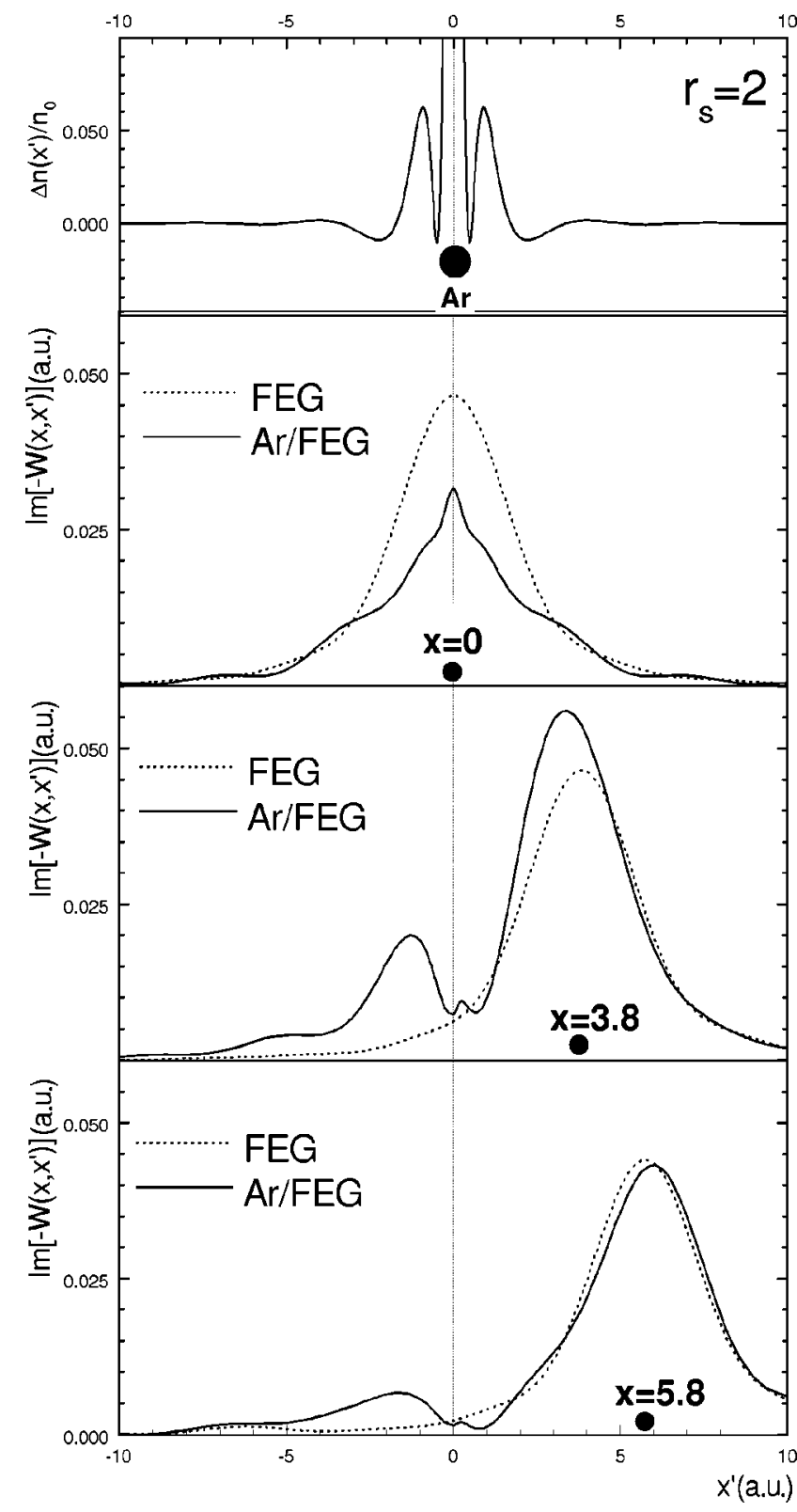

FIG. 1. Top panel: electronic density (in units of the background electronic density $n_{0}$ ) induced in the valence-band $\Delta n=n-n_{0}$ (where $n$ is the valence band electronic density) by an Ar atom embedded in a free electron gas of $r_{s}=2$. Lower panels (from top to bottom, magnitudes in atomic units): imaginary part of the screened interaction $\operatorname{Im}\left[-W\left(x, x^{\prime}, \omega\right)\right]$ as a function of $x^{\prime}$, and for different values of $x(x=0, x=3.8$, and $x=5.8)$. The Ar atom is located at the origin of the coordinate system. The solid line is the result including the Ar impurity in the calculation, and the dotted line is the unperturbed electron gas result. The energy is $\omega$ $=0.17 \omega_{p}$.

tions are always created inside the surface, and the maximum value of $\operatorname{Im}\left[-W\left(x, x^{\prime}, \omega\right)\right]$ does not necessarily correspond to the position $x=x^{\prime} .^{21}$

Now we consider a localized external perturbation around the impurity: the density fluctuation of electronic charge, $\rho^{\text {ext }}(\mathbf{r})=\psi_{\alpha}^{*}(\mathbf{r}) \psi_{\mathbf{k}_{1}}(\mathbf{r})$, which accompanies the decay of one electron from a valence-band state $\psi_{\mathbf{k}_{1}}(\mathbf{r})$ to a bound state of 

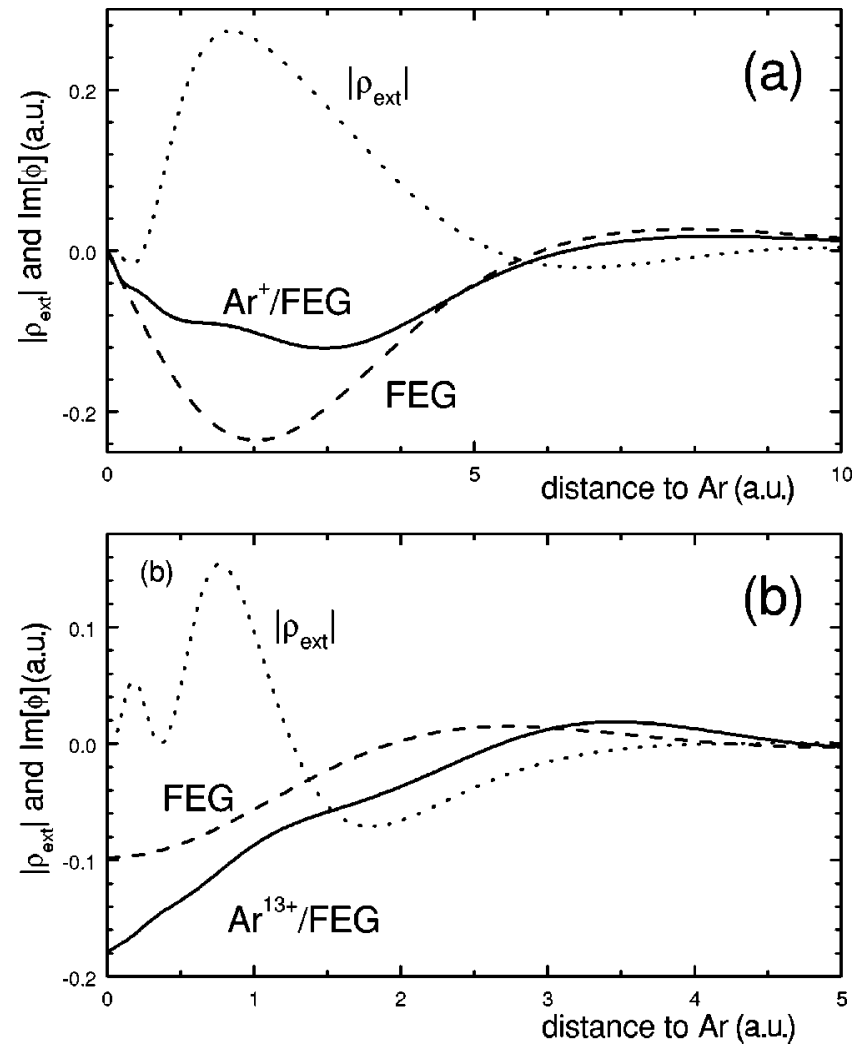

FIG. 2. Modulus of the external density $\left|\rho_{\text {ext }}(r)\right|$ (dotted line) and $r$ times the imaginary part of the potential $\operatorname{Im}[\phi(r, \omega)]$ as a function of the distance from the ion. The process is the decay of an electron at the Fermi level into the $3 p$ state of (a) an $\mathrm{Ar}^{+}$ion, and (b) an $\mathrm{Ar}^{13+}$ ion. The density parameter is $r_{s}=2$. The calculation of the response function includes (solid line) or excludes (dashed line) the Ar ions. Only the dominant partial-wave components [continuum $d$ electrons $\left(l_{1}=2\right)$ for (a) and continuum $p$ electrons $\left(l_{1}\right.$ $=1$ ) for (b)] are shown. All magnitudes are in atomic units.

an ion, $\psi_{\alpha}(\mathbf{r})$. This inelastic process corresponds to the filling of a hole in the ion. We remind the reader at this point that the external perturbation that we treat in linear response is $\rho^{\text {ext }}(\mathbf{r})$ and not the embedded impurity. The latter is considered as part of the system, and thus fully included (formally to all orders of perturbation theory) in the calculation. The calculation of the transition rate for this process is performed after expanding all quantities in the sphericalharmonic basis set. Assuming spherical symmetry, and fixing the angular momentum of the final bound state $\psi_{\alpha}(\mathbf{r})$ to $l_{\alpha}$, the transition rate per unit time and per unit of momentum modulus $k_{1}\left(\epsilon_{1}=k_{1}^{2} / 2\right)$ is obtained as a sum over different angular momentum components: ${ }^{22}$

$$
\frac{d \Gamma}{d k_{1}}=k_{1}^{2} \sum_{l, l_{1}} \zeta\left(l, l_{1}, l_{\alpha}\right) \operatorname{Im}\left\{\int d r r^{2}\left[\rho_{\mathrm{ext}}^{l_{1}}(r)\right]^{*} \phi^{l l_{1}}(r, \omega)\right\},
$$

where $\omega=\epsilon_{1}-\epsilon_{\alpha}$,

$$
\zeta\left(l, l_{1}, l_{\alpha}\right)=(2 l+1)\left(2 l_{1}+1\right)\left(\begin{array}{ccc}
l l_{1} l_{\alpha} \\
0 & 0 & 0
\end{array}\right)^{2}
$$

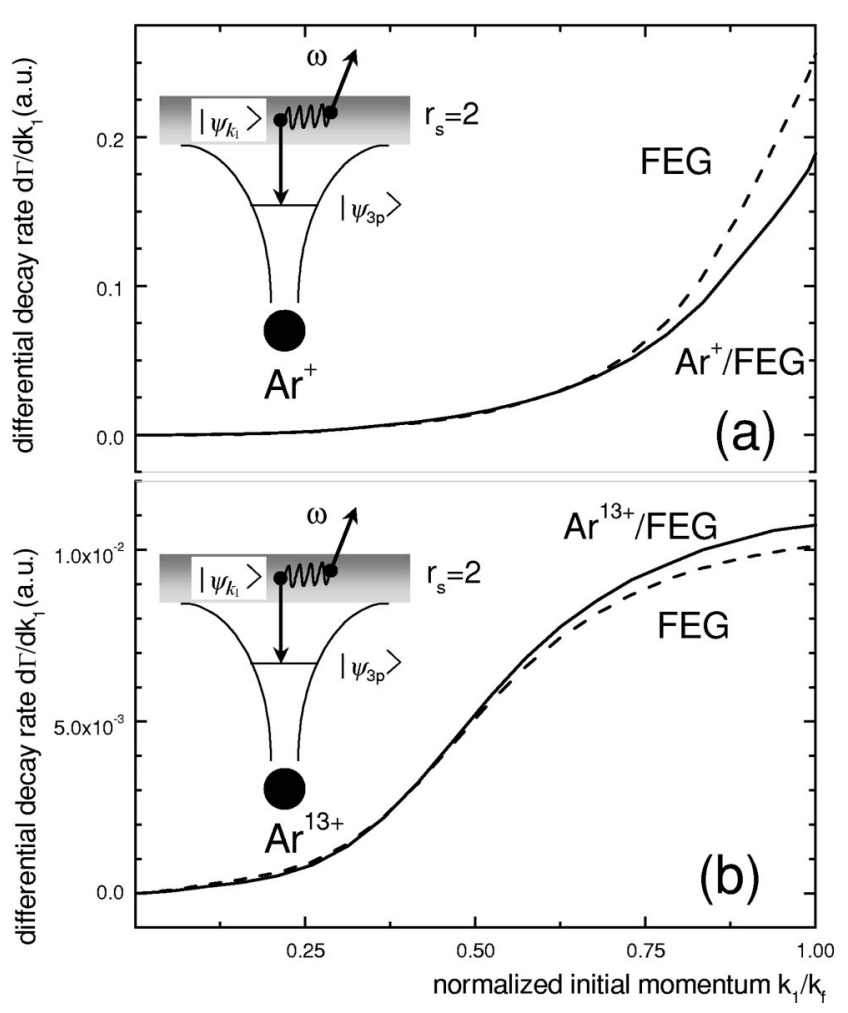

FIG. 3. Differential transition rate $d \Gamma / d k_{1}$ (in atomic units) as a function of the initial momentum of the electron (normalized to the Fermi momentum) $k_{1} / k_{f}$ for a decay process from a free-electron gas of $r_{s}=2$ to the $3 p$ state of (a) an $\mathrm{Ar}^{+}$ion and (b) an $\mathrm{Ar}^{13+}$ ion. The line styles follow the same convention of Fig. 2. A schematic drawing of the decay process is shown in the inset.

is a coefficient resulting from the analytical integration of the angular part, $l_{1}$ is the angular momentum component of the initial state wave function, and $l$ is associated with the $l$-multipole term in the expansion of the screened interaction $W\left(\mathbf{r}, \mathbf{r}^{\prime}, \omega\right) . \phi^{l l_{1}}(r, \omega)$ are partial-wave components in the expansion of the self-consistent potential $\phi(\mathbf{r}, \omega)$, defined as

$$
\phi(\mathbf{r}, \omega)=\int d \mathbf{r}^{\prime} \rho^{\operatorname{ext}}\left(\mathbf{r}^{\prime}\right) W\left(\mathbf{r}, \mathbf{r}^{\prime}, \omega\right)
$$

We study two regimes: low $\left(\omega \approx 0.9 \omega_{p}\right)$ and high $(\omega$ $\left.\approx 2.8 \omega_{p}\right)$ transition energies. They correspond to the capture of a $3 p$ electron from the Fermi level by $\mathrm{Ar}^{+}$and $\mathrm{Ar}^{13+}$, respectively. The electronic configuration of the $\mathrm{Ar}^{13+}$ ion is $1 s^{1} 3 s^{2} 3 p^{2}$. We show in Fig. 2 the imaginary part of the self-consistent potential $\operatorname{Im}\left[\phi^{l l_{1}}(r, \omega)\right]$ generated by the density fluctuation $\rho_{\text {ext }}(r)$ that is associated with the 3 p capture by $\mathrm{Ar}^{+}$[Fig. 2(a)] and $\mathrm{Ar}^{13+}$ [Fig. 2(b)]. The radial dependence of $\left|\rho_{\text {ext }}^{l_{1}}(r)\right|$ is plotted as well. The localization of $\left|\rho_{\text {ext }}^{l_{1}}(r)\right|$ in the vicinity of the ion determines the range of distances, which is important to calculate the transition rate of the process. Only the dominant partial-wave contribution to the process is shown: $l=1$ and $l_{1}=2$ for $\mathrm{Ar}^{+}$(i.e., $d$ electrons from the valence band that undergo a dipolar transition) and $l=0$ and $l_{1}=1$ for $\operatorname{Ar}^{13+}$ ( $p$ electrons that un- 
dergo a monopolar transition). Results using two different levels of approximation in the calculation of the response function $\chi\left(\mathbf{r}, \mathbf{r}^{\prime}, \omega\right)$ (with and without the Ar ion) are plotted in Fig. 2.

The piling up of charge in the vicinity of the ion has important consequences in modifying the screening properties, as well as the excitation spectrum of the system. Both effects are important in determining the electron dynamics and the transition rate, although their role is different at low and high $\omega$. At low $\omega$, enhancement of the screening reduces the strength of the Coulomb interaction in such a way that the imaginary part of the screened interaction is significantly reduced. However, at high $\omega$, screening is not so efficient, and there is a net increase (instead of a reduction), in the value of the imaginary part of the screened interaction due to the local increase of available electrons near the impurity.

Capture rates from the valence band to bound states of the ion are calculated by integrating the external density and the self-consistent potential using Eq. (4). The results of the differential probability as a function of the initial momentum of the electron are plotted in Fig. 3 for the same two systems of Fig. 2. Perturbation of the medium by the ion introduces an increase of roughly $20 \%$ in the value of the rate $\Gamma$ for the low energy range [Fig. 3(a)], and a reduction of 5-10\% for higher excitation energies [Fig. 3(b)].

In summary, we have developed a general method to calculate the self-consistent response function of an impurity embedded in a FEG. We have applied our method to study the modifications introduced by the presence of an impurity in the dynamic screening of the medium electrons. We have considered the case of an atomic impurity, but our conclu- sions would be similar for other kind of impurities (core holes, molecules, etc.). We have shown that the localization of screening charge induced by the impurity in its vicinity strongly modifies electron dynamics through a change in the electron-electron interaction strength and in the probability of electron excitation. The relative weight of these two effects depends on the particular value of the excitation energy $\omega$. In simple terms, one could say that as far as determining the imaginary part of the screened interaction is concerned, the electron-electron screening effect is more important for low $\omega$, while the increase in the available number of excitations (modification of phase space) is more importnt at high $\omega$. The nontrivial balance between these two effects makes difficult to predict a priori the influence of the impurity on the time scales of inelastic transitions that depend on the electron-electron interaction in its vicinity. As an example, we have calculated the decay rate of valence-band electrons to the $3 p$ bound state of Ar, for which the final rate is shown to be appreciably modified by the impurity perturbation. This modification is not expected to be specific of this particular case, but can be generalized to other inelastic processes that depend on electron-electron interactions and are highly localized in space.

We are grateful to M. A. Cazalilla and E. Zaremba for fruitful discussions. This work was supported in part by the Basque Departamento de Educación, Universidades e Investigación, the University of the Basque Country UPV/EHU (Grant No. 9/UPV 00206.215-13639/2001), and the Spanish Ministerio de Ciencia y Tecnología (Grant No. MAT20010946). R.D.M. acknowledges financial support by the Gipuzkoako Foru Aldundia.
${ }^{1}$ A. Zangwill and P. Soven, Phys. Rev. A 21, 1561 (1980).

${ }^{2}$ M.J. Stott and E. Zaremba, Phys. Rev. A 21, 12 (1980).

${ }^{3}$ F. Grimaldi, A. Grimaldi-Lecourt, and M.W.C. Dharma-wardana, Phys. Rev. A 32, 1063 (1985).

${ }^{4}$ F. Perrot and M.W.C. Dharma-wardana, Phys. Rev. A 41, 3281 (1990).

${ }^{5}$ W. Ekardt, Phys. Rev. Lett. 52, 1925 (1984).

${ }^{6}$ A. Rubio, L.C. Balbás, and J.A. Alonso, Phys. Rev. B 45, 13657 (1992)

${ }^{7}$ M.J. Puska and R.M. Nieminen, Phys. Rev. A 47, 1181 (1993).

${ }^{8}$ S. Grabowski, M.E. Garcia, and K.H. Bennemann, Phys. Rev. Lett. 72, 3969 (1994).

${ }^{9}$ K. Sturm, E. Zaremba, and K. Nuroh, Phys. Rev. B 42, 6973 (1990).

${ }^{10}$ E. Zaremba and K. Sturm, Phys. Rev. Lett. 66, 2144 (1991).

${ }^{11}$ A. Zangwill and D.A. Liberman, Phys. Rev. B 36, 6705 (1987).

${ }^{12}$ N. Lorente and R. Monreal, Phys. Rev. B 53, 9622 (1996).

${ }^{13}$ M.A. Cazalilla, N. Lorente, R. Díez Muiño, J.-P. Gauyacq, D.
Teillet-Billy, and P.M. Echenique, Phys. Rev. B 58, 13991 (1998).

${ }^{14}$ A. Yazdani, C.M. Howald, C.P. Lutz, A. Kapitulnik, and D.M. Eigler, Phys. Rev. Lett. 83, 176 (1999).

${ }^{15}$ A.G. Borisov, A.K. Kazansky, and J.P. Gauyacq, Phys. Rev. B 64, 201105 (2001); 65, 205414 (2002).

${ }^{16}$ A.M. Ermolaev and B. Haghighi, Low Temp. Phys. 25, 446 (1999).

${ }^{17}$ J. Li, W.-D. Schneider, R. Berndt, and B. Delley, Phys. Rev. Lett. 80, 2893 (1998).

${ }^{18}$ V. Madhavan, W. Chen, T. Jamneala, M.F. Crommie, and N.S. Wingreen, Science 280, 567 (1998).

${ }^{19}$ W. Kohn and L.J. Sham, Phys. Rev. 140, A1133 (1965).

${ }^{20}$ O. Gunnarson and B.I. Lundqvist, Phys. Rev. B 13, 4274 (1976).

${ }^{21}$ P.M. Echenique, J.M. Pitarke, E.V. Chulkov, and A. Rubio, Chem. Phys. 251, 1 (2000).

${ }^{22}$ A. Liebsch, Electronic Excitations at Metal Surfaces, (Plenum, New York, 1997). 